\title{
Administração Vaginal do Gel de Allopregnanolona a Mulheres Menopausadas em Uso de
} Estrogenioterapia

\author{
Autora: Paula Andrea de Albuquerque Salles Navarro \\ Orientador: Prof. Dr. Rui Alberto Ferriani
}

Dissertação de Mestrado apresentada a área de Tocoginecologia da Faculdade de Medicina de Ribeirão Preto - USP, em 31 de julho de 2000.

\begin{abstract}
Objetivos: Avaliar a tolerabilidade e obter dados preliminares referentes aos efeitos endometriais da allopregnanolona, administrada sob a forma de gel, por via vaginal, a pacientes na pós-menopausa em uso de estrogenioterapia oral, ao longo de 2 ciclos de tratamento, assim como obter dados preliminares do efeito da droga de estudo, nos niveis séricos de gonadotrofinas, estradiol e progesterona.
\end{abstract}

Pacientes e métodos: Foram incluídas no presente estudo 13 pacientes na pós-menopausa, divididas em 2 grupos: grupo 1: primeiras 7 pacientes incluidas no estudo (mediana de idade $=52$ anos; mediana de tempo de amenorréia $=2$ anos ) e grupo 2: 6 últimas pacientes (mediana de idade $=55,5$ anos; mediana de tempo de amenorréia $=4,3$ anos ). Todas as pacientes foram submetidas a 2 ciclos consecutivos de tratamento, cada qual com 28 dias de duração, utilizando $2 \mathrm{mg}$ ao dia de valerato de estradiol, por via oral, continuamente, associado ao gel de allopregnanolona a $8 \%$, por via vaginal, nos 10 últimos dias de um único ciclo (grupo 1) ou dos 2 ciclos (grupo 2). Todas as pacientes foram avaliadas quanto à caracterização dos efeitos adversos e sangramento genital. Coleta de sangue para dosagens hormonais e biópsias de endométrio foram realizadas no $28^{\circ}$ e no $56^{\circ}$ dia de tratamento, nas pacientes do grupo $1 \mathrm{e}$, apenas, no $56^{\circ}$, nas do grupo 2 .

Resultados: A taxa de aderência ao tratamento foi de $100 \%$ nas pacientes de ambos os grupos. A mastalgia foi o efeito adverso mais freqüente (4 casos), seguido pela cefaléia e pela dor abdominal ( 2 casos cada), todos estes relacionados ao uso do valerato de estradiol. Uma paciente referiu prurido vulvar durante o uso do gel de allopregnanolona. Todos os efeitos adversos fo- ram leves e não interferiram na utilização das medicações prescritas. Não houve diferença significativa $(\mathrm{P}$ $=1,0$ ) entre as proporções de sangramento genital por deprivação hormonal após um $(23,1 \%)$ ou dois ciclos de utilização da allopregnanolona (33,3\%). Não encontramos diferença significativa $(\mathrm{P}=0,27)$ entre as proporções de endométrio secretor e proliferativo após um $(57,1 \%$ e $42,9 \%$, respectivamente) ou dois ciclos $(16,7 \%$ e $66,6 \%$ ) de uso da allopregnanolona. Contudo há uma aparente redução da percentagem de endométrio secretor após 2 meses de utilização da allopregnanolona, quando comparada a um único ciclo de uso desta droga. Não houve diferença estatisticamente significante entre os niveis séricos de FSH, LH, estradiol ou progesterona após um ciclo com valerato de estradiol exclusivamente, quando comparados a um ou dois ciclos de uso da allopregnanolona.

Conclusões: Observamos boa tolerabilidade à administração vaginal do gel de allopregnanolona a mulheres na pós-menopausa, em uso de estrogenioterapia oral com valerato de estradiol, refletida pela baixa incidência de efeitos adversos e pela boa aceitabilidade à terapêutica. Aparentemente, a allopregnanolona não interfere nos niveis séricos de gonadotrofinas, estradiol e progesterona. Estudos com maiores casuística e tempo de seguimento são necessários para se determinar os efeitos endometriais desta nova droga, e, conseqüentemente, da sua possivel utilização futura, nos diversos esquemas de terapia de reposição hormonal vigentes.

Palavras-chave: Terapia de reposição hormonal. Endométrio: avaliação. Mastalgia. Menopausa.

\section{Estudo Anatomoclínico da Expressão do p53 no Adenocarcinoma de Ovário}

Autora: Patrícia Morais Martins

Orientador: Prof. Dr. Paulo Traiman

Dissertação de Mestrado apresentada na Faculdade de Medicina de Botucatu - UNESP (Programa de Pós-Graduação em Ginecologia e Obstetrícia, área de concentração em Ginecologia), em 31 de janeiro de 2000.

O câncer de ovário continua sendo uma entidade letal e silenciosa nestes últimos 40 anos, apesar dos avanços diagnósticos e terapêuticos. A literatura destacou intensamente o papel de vários marcadores tumorais no estudo do carcinoma ovariano. Estudamos neste trabalho a freqüência da alteração da proteína p53 no carcinoma de ovário e sua correlação com as variedades histológicas e com os parâmetros clínicos. Avaliamos também a correlação do comportamento biológico nas pacientes com expressão do p53. Analisamos, retrospectivamente, 41 pacientes através de dados clínicos e anatomopatológicos, correlacionando com a expressão do p53. A idade variou de 21 a 81 anos (mediana de 50 anos). Observamos $25 \%$ das pacientes estádio I, 7,5\% estádio II, 45\% estádio III, e 22,5\% estádio IV. Foram consideradas inoperáveis 7 pacientes (17,07\%). O p53 mostrou-se positivo em $45,9 \%$ dos casos. Realizamos associação entre vários parâmetros clínicos e anatomopatológicos e encontramos significado estatístico entre a presença do p53 e a taxa de sobrevivência.

Palavras-chave: Ovário: câncer. Oncogene. 


\title{
Toxoplasmose: Soroprevalência em Puérperas Atendidas pelo SUS em Cuiabá, Mato Grosso
}

Autor: Paulo Roberto Dutra Leão

Orientador: Prof. Dr. Sebastião Freitas de Medeiros

Co-orientador: Prof. Dr. José Meirelles Filho

Dissertação de Mestrado apresentada ao curso de pós-graduação do Instituto de Saúde Coletiva da Universidade Federal de Mato Grosso, em 27 de julho de 2001, para obtenção do título de mestre em Saúde e Ambiente.

A toxoplasmose é uma doença parasitária, causada pelo protozoário Toxoplasma gondii, geralmente benigna, exceto na infecção fetal, transmitida verticalmente pela gestante, quando pode cursar como uma doença devastadora para o concepto. O risco de adquirir a doença durante a gestação está correlacionado à sua prevalência na população, que é muito variável nas diferentes regiões do mundo (10 a 80\%). O objetivo deste estudo foi estabelecer a taxa de soroprevalência contra Toxoplasma gondii em puérperas atendidas pelo SUS, em duas maternidades de Cuiabá, correlacionando os resultados com a exposição a fatores de risco geralmente associados a esta doença. Trata-se de um estudo de corte transversal, incluindo 205 pacientes com idade entre 14 e 43 anos, em puerpério imediato, atendidas nestes hospitais durante dois meses. As pacientes foram entrevistadas e submetidas a coleta de amostras de sangue para dosagem de imunoglobu- lina $\mathrm{G}$ contra toxoplasma, através de um método imunoenzimático (MEIA). A idade média das pacientes foi de 22,4 anos e a maioria estava entre a primeira e segunda gestações. A soropositividade para toxoplasma foi detectada em 165 (70,7\%) das 205 pacientes estudadas. Esta prevalência é concordante com muitos estudos nacionais e de países em desenvolvimento. Analisando-se os fatores de risco para a contaminação pelo toxoplasma, encontrou-se associação, estatisticamente significativa, com o convivio com gato $(p=0,001)$ e manipulação e/ou ingestão de carne crua $(p=0,04)$. Não se identificou associação com a idade das pacientes, história de abortamentos anteriores e manuseio do solo. A grande maioria das pacientes $(78,1 \%)$ desconhecia a existência da toxoplasmose.

Palavras-chave: Toxoplasmose. Infecções na gravidez. Soroprevalência.

\section{Estudo Morfométrico do Epitélio e do Estroma Mamário sob Tratamento com Anticoncepcional} Hormonal Combinado Oral

\author{
Autora: Nara Fabricia de Mattia \\ Orientador: Prof. Dr. Afonso Celso Pinto Nazário
}

Dissertação de Mestrado apresentada à Universidade Federal de São Paulo - Escola Paulista de Medicina, em 2 de maio de 2001.

Foram estudadas 20 pacientes com fibroadenoma diagnosticado clinicamente e por punção aspirativa com agulha fina. As que desejavam usar anticoncepcional hormonal combinado oral (10 pacientes), o fizeram por quatro ciclos consecutivos, com um agente composto de lenorgestrel $(0,15)$ e etinilestradiol $(0,03 \mathrm{mg})$. Propôs-se comparar a morfometria do epitélio e do estroma da mama normal das usuárias de anticoncepcional (estudo ou A) e das não-usuárias (controle ou B), na fase lútea. Retirou-se o fibroadenoma (que demonstrou ser benigno) e efetuou-se biópsia a $1 \mathrm{~cm}$ da lesão para retirada de fragmento de mama normal dos dois grupos. O tecido foi fixado em formol a $10 \%$, cortado, colocado em lâminas, corado pela $\mathrm{HE}$ e avaliado pelo sistema de análise digital de imagem, que emprega um microscópico ótico acoplado a uma videocâmera colorida que transmite a imagem a um microcomputador dotado de placa digitalizadora, com aumento de 400 vezes. Avaliou-se o volume neclear do epitélio dos ductos terminais e do estroma, bem como o índice mitótico. No tocante ao volume nuclear do epitélio dos ductos terminais, a média do grupo de estudo (A) foi 142,6 $\mu^{3} \mathrm{~m}^{3}$ (desvio padrão de $30,9 \mu \mathrm{m}^{3}$ ) e, no controle (B), foi $130,9 \mu \mathrm{m}^{3}$ (desvio padrão de $46,8 \mu \mathrm{m}^{3}$. Não demonstrou diferença significativa $(p=0,52)$. No estroma a média do volume nuclear do grupo (A) foi $26,1 \mu \mathrm{m}^{3}$ (desvio padrão de 8,3) e, no controle (B), foi $21,5 \mu \mathrm{m}^{3}$ (desvio padrão de 13,1 ). Não houve diferença significativa entre os grupos $(\mathrm{p}=0,37)$. A média do índice mitótico de epitélio dos ductos terminais do grupo estudo foi $19,5 \%$ (desvio padrão de 20) e, no controle, $17,2 \%$ (desvio padrão 18); também não houve diferença significativa $(\mathrm{p}=0,14)$. Assim, concluí-se não haver diferença significante na morfometria do epitélio e do estroma entre mama normal de não-usuárias na fase lútea e de usuárias de quatro ciclos de anticoncepcional hormonal oral composto de levonorgestrel $(0,15 \mathrm{mg})$ e etinilestradiol $(0,03 \mathrm{mg})$.

Palavras-chave: Mama normal. Epitélio mamário. 